\title{
PRL-3 promotes the peritoneal metastasis of gastric cancer through the PI3K/Akt signaling pathway by regulating PTEN
}

\author{
JIANBO XIONG $^{1 *}$, ZHENGRONG LI $^{1}{ }^{*}$, YANG ZHANG $^{1 *}$, DAOJIANG LI ${ }^{1}$, GUOYANG ZHANG $^{1}$, XIANSHI LUO $^{1}$, \\ ZHIGANG JIE $^{1}$, YI LIU ${ }^{1}$, YI CAO ${ }^{1}$, ZHIBIAO LE ${ }^{1}$, SHENGXING TAN ${ }^{1}$, WENYU ZOU ${ }^{1}$, PEITAO GONG ${ }^{1}$, \\ LINGYU QIU ${ }^{2}$, YUANYUAN LI ${ }^{2}$, HUAN WANG ${ }^{2}$ and HEPING CHEN ${ }^{2}$ \\ ${ }^{1}$ Department of Gastrointestinal Surgery, The First Affiliated Hospital, Nanchang University, Nanchang, Jiangxi 330000; \\ ${ }^{2}$ The Key Laboratory of Basic Pharmacology, School of Pharmaceutical Science, \\ Nanchang University, Nanchang, Jiangxi 330006, P.R. China
}

Received March 10, 2016; Accepted May 17, 2016

DOI: $10.3892 /$ or.2016.5030

\begin{abstract}
Peritoneal metastasis is the most frequent cause of death in patients with advanced gastric carcinoma (GC). The phosphatase of regenerating liver-3 (PRL-3) is recognized as an oncogene and plays an important role in GC peritoneal metastasis. However, the mechanism of how PRL-3 regulates GC invasion and metastasis is unknown. In the present study, we found that PRL-3 presented with high expression in GC with peritoneal metastasis, but phosphatase and tensin homologue (PTEN) was weakly expressed. The p-PTEN/PTEN ratio was also higher in GC with peritoneal metastasis than that in the normal gastric tissues. We also found the same phenomenon when comparing the gastric mucosa cell line with the GC cell lines. After constructing a wild-type and a mutant-type plasmid without enzyme activity and transfecting them into GC SGC7901 cells, we showed that only PRL-3 had enzyme activity to downregulate PTEN and cause PTEN phosphorylation. The results also showed that PRL-3 increased the expression levels of MMP-2/MMP-9 and promoted the migration and invasion of the SGC7901 cells. Knockdown of PRL-3 decreased the expression levels of MMP-2/MMP-9 significantly, which further inhibited the migration and invasion of the GC cells. PRL-3 also increased the expression ratio of p-Akt/Akt, which indicated that PRL-3 may mediate the PI3K/
\end{abstract}

Correspondence to: Professor Zhengrong Li, Department of General Surgery, The First Affiliated Hospital, Nanchang University, 17 Yongwai Zheng Road, Nanchang, Jiangxi 330006, P.R. China E-mail: lzr13@foxmail.com

Professor Heping Chen, The Key Laboratory of Basic Pharmacology School of Pharmaceutical Science, Nanchang University, Nanchang, Jiangxi 330006, P.R. China

E-mail: chenheping69@hotmail.com

${ }^{*}$ Contributed equally

Key words: PRL-3, PTEN, phosphorylation, PI3K/Akt pathway, gastric cancer
Akt pathway to promote GC metastasis. When we transfected the PTEN siRNA plasmid into the PRL-3 stable low expression GC cells, the expression of p-Akt, MMP-2 and MMP-9 was reversed. In conclusion, our results provide a bridge between PRL-3 and PTEN; PRL-3 decreased the expression of PTEN as well as increased the level of PTEN phosphorylation and inactivated it, consequently activating the PI3K/Akt signaling pathway, and upregulating MMP-2/MMP-9 expression to promote GC cell peritoneal metastasis.

\section{Introduction}

Gastric cancer (GC) remains the third leading cause of cancer-related death in both genders worldwide and causes over 723,000 deaths worldwide per year $(1,2)$. Even with the improvement in diagnostic techniques and advancement in therapeutic modalities, the 5-year survival rates among patients are still disappointing, and one of the predominant causes is metastasis (3-5). Peritoneal metastasis, as a common mode of metastasis for advanced GC and a common feature of the natural history of GC, is the most frequent cause of death in patients with advanced GC (6). It is necessary to investigate the molecular mechanisms of GC metastasis and to develop new strategies to prevent GC peritoneal metastasis.

Phosphatase of regenerating liver-3 (PRL-3), a member of the PRL family of protein tyrosine phosphatases, plays an important role in GC metastasis (7). Researchers have demonstrated that PRL-3 induces cancer-associated phenotypes including proliferation, migration, invasion, tumorigenesis and metastasis (8-11), which also arise in GC (12). There are also various experiments showing that the catalytic activity of PRL-3 is directly involved in tumor invasion, metastasis and tumor angiogenesis $(10,13)$. In our previous study, we found that in primary GC with peritoneal metastasis, PRL-3 expression was significantly higher than that in the corresponding primary GC and PRL-3 expression was negatively correlated with prognosis in GC patients (14). In addition, various studies have also indicated that upregulation of PRL-3 expression correlates with GC invasion and metastasis (15-17). Furthermore, in our previous study, we also found that miR-495 and miR-551a both act as tumor-suppressors by targeting the PRL-3 oncogene 
and inhibiting GC cell migration and invasion (18). However, the downstream mechanism by which the signaling pathway facilitates PRL-3-promoted motility, invasion and metastasis of GC cells remains unknown, and warrants further research.

Phosphatase and tensin homologue (PTEN; deleted on chromosome 10) is a tumor-suppressor gene featuring dual-specificity phosphatase activities $(19,20)$. It plays crucial roles in maintaining normal cell activities and functions $(19,20)$. PTEN is a lipid phosphatase that catalyzes the conversion of phosphatidylinositol-3,4,5,-trisphosphate (PIP3) to phosphatidylinositol-4,5-bisphosphate (PIP2) (21), which can induce apoptosis and suppress proliferation. Loss of PTEN function leads to excessive PIP3 accumulation at the plasma membrane and subsequent derepression of the phosphatidylinositol-3-kinase/protein kinase B (PI3K)/Akt pathway, which in turn stimulates cell growth, proliferation, survival and other cellular processes $(22,23)$.

The PI3K/Akt signaling pathway is an important driver of cell proliferation and survival (24). PI3K signaling has been demonstrated to be involved in GC progression, including cell growth, metabolism, survival, metastasis and resistance to chemotherapy (25-28). PRL-3 most likely lies upstream of PI3K, as treatment with the PI3K inhibitor LY294002 abrogated PRL-3-mediated AKT activation (29,30). Additionally, PRL-3 overexpression downregulated the expression of PTEN in DLD-1 colorectal carcinoma (29) and melanoma cells (31). Since PTEN is the most important negative regulator of the PI3K/Akt pathway, PTEN downregulation may be an important means of PRL-3-driven PI3K/Akt signaling, although PI3K activation by other mechanisms may exist.

Before cancer cells metastasize to distant organs, they must disseminate from the site of the primary tumor (32). Extracellular matrix degradation is an essential step in tumor invasion and metastasis. Matrix metalloproteinases (MMPs), including MMP-2 and MMP-9, are capable of cleaving cell-surface proteins as well as degrading components of the extracellular matrix, allowing migratory cells to invade neighboring tissues and break through the basement membrane (33). In a previous study, PRL-3 was found to promote the motility, invasion and metastasis of LoVo colon cancer cells through PRL-3-integrin $\beta 1$-ERK1/2 and -MMP2 signaling (34). PRL-3 also enhanced MMP-2 secretion and cellular invasiveness via activation of mTOR in the human colon cancer HCT116 cell line (35).

In consideration of the crucial role of PRL-3 in GC metastasis and the emerging character of PTEN in GC, we speculated that PRL-3 regulates the expression or post-translational modification of PTEN and then activates the PI3K/Akt signaling pathway, upregulating MMP-2/MMP-9 expression, further to promote GC peritoneal metastasis by its phosphatase enzyme activity. In the present study, we described that PRL-3 was overexpressed in GC with peritoneal metastasis and in GC lines with high enzyme activity, while PTEN was weakly expressed in GC with peritoneal metastasis and in GC cell lines. The present study also showed that only PRL-3 had the enzyme activity to downregulate the expression of PTEN and increased the ratio of p-PTEN/PTEN. We also found that PRL-3 activated the PI3K/Akt signaling pathway and further upregulated the expression of MMP-2 and MMP-9 to promote $\mathrm{GC}$ peritoneal metastasis.

\section{Materials and methods}

Tissue samples. All human gastric tissue samples including 21 specimens of normal gastric mucosa, 49 specimens of GC without peritoneal metastasis and 23 specimens of GC with peritoneal metastasis were obtained from the Department of General Surgery of The First Affiliated Hospital of Nanchang University (Nanchang, China). All specimens were immediately frozen in liquid nitrogen and were stored at $-80^{\circ} \mathrm{C}$ until further use. Informed consent was obtained from all patients, and the present study was approved by the institutional review committee. None of the patients received radiotherapy or chemotherapy before the operation. Histological diagnosis was confirmed for each specimen. All of the samples were obtained with informed consent for molecular analysis and were approved by the Ethics Committee of the The First Affiliated Hospital of Nanchang University.

Cell culture. The human gastric mucosal cell line GES-1 was preserved in our central laboratory. Human gastric adenocarcinoma cancer cell lines SGC7901, MKN28, MGC803, AGS and MKN45 were obtained from the Type Culture Collection of the Chinese Academy of Sciences (Shanghai, China). Cells were cultured at $37^{\circ} \mathrm{C} / 5 \% \mathrm{CO}_{2}$ in Dulbecco's modified Eagle's medium (DMEM; Biological Industries, Israel), supplemented with $10 \%$ fetal bovine serum (FBS) (MRC, China), $100 \mathrm{U} / \mathrm{ml}$ penicillin and $100 \mu \mathrm{g} / \mathrm{ml}$ streptomycin.

Construction of a wild-type plasmid EGFP-PRL-3 and a mutant-type plasmid EGFP-PRL-3 (D72A/C104S) and transfection into SGC7901 cells. We constructed a wild-type plasmid EGFP-PRL-3 with the enzyme activity of PRL-3 and a mutant-type plasmid EGFP-PRL-3 (D72A/C104S) containing an inactivating mutation of the essential catalytic cytokine residue to serine at position 104 in the phosphate active site according to related studies $(13,36,38)$. All of the vectors were designed and synthesized by GenePharma Corporation (Shanghai, China). After being confirmed by DNA sequencing, the EGFP-PRL-3 and the EGFP-PRL-3 (D72A/C104S) vectors were transfected into SGC7901 cells using Lipofectamine 2000 reagent (Thermo Fisher Scientific, Inc., Waltham, MA, USA) according to the manufacturer's protocol. After screening, the SGC7901 cancer cells stably expressed PRL-3; the wild-type EGFP-PRL-3 with the enzyme activity was the wild-type group and the mutant-type EGFP-PRL-3 (D72A/C104S) was the mutant-type without enzyme activity. The enzyme activity of PRL-3 was detected by a phosphate activity detection kit (Beyotime, Shanghai, China/Invitrogen, Carlsbad, CA, USA) according to the manufacturer's instructions and confirmed that the two groups met the requirements (39-42).

Construction of the EGFP-PRL-3 and PRL-3-RNAi plasmids and construction of stable cell lines. To construct the EGFP-PRL-3 plasmid, human PRL-3 was amplified by RT-PCR using the primers: 5'-TACCGGACTCAGATCTCGA GCGCCACCATGGCTCGGATGAACCGC-3' and 5'-GATC CCGGGCCCGCGGTACCGTCATAACGCAGCACCGGGT CT-3' and was cloned into the GV230 vector. To acquire the generation of PRL-3 knockdown in the SGC7901 cells, we 
constructed three candidate human PRL-3 siRNA target sequences and a blank plasmid control vector. All of the vectors were designed and synthesized by GeneChem Corporation (Shanghai, China). The PRL-3 shRNA target sequences were as follows: 5'-GCTCACCTACCTGGAGA AA-3' [PRL-3-RNAi (7330)], 5'-ACAGAGGCTGCGGTTCA AA-3' [PRL-3-RNAi (7331)], 5'-CCTGTTCTCGGCACCT TAA-3' [PRL-3-RNAi (7332)], 5'-GGATCTCGTTCTCCTC ATT-3' [PRL-3-RNAi (7333)]. All the vectors were transfected into SGC7901 cells using Lipofectamine 2000 reagent according to the manufacturer's protocol. We selected PRL-3-RNAi (7332) with the highest efficacy as the suitable vector after evaluating the interference efficiency by western blotting. After screening, we constructed the stable expression cell lines. The SGC7901/EGFP-PRL-3 cell line was the PRL-3 high expression group and the SGC7901/PRL-3-RNAi cell line was the PRL-3 low expression group.

Construction of the PTEN-knockdown plasmid and transfection into the PRL-3 low expression cell line. For the RNA interference of human PTEN, three specific small interfering RNAs were used. The shRNA target sequences were: 5'-GCGCTATGTGTATTATTAT-3' [PTEN-RNAi (17260)], 5'-ACAGCTAGAACTTATCAAA-3' [PTEN-RNAi (17261)], 5'-AGAACTTATCAAACCCTTT-3' [PTEN-RNAi (17262)], 5'-TGCAGATAATGACAAGGAA-3' [PTEN-RNAi (17263)], respectively. All of the vectors were designed and synthesized by GeneChem. All of the vectors were transfected into SGC7901 cells using Lipofectamine 2000 reagent. After screening, we constructed the stable expression cell lines and selected the high interfering efficiency PTEN-RNAi (17261) group.

Quantitative real-time RT-PCR analyses. The detailed steps of RNA extraction and qRT-PCR were described in our previous study (18). TRIzol reagent was purchased from Invitrogen. Applied Biosystems 7500 Fast Real-Time PCR System and SYBR-Green PCR Master Mix were purchased from Applied Biosystems (Foster City, CA, USA), Taq PCR Master Mix was purchased from TransGen (Beijing, China). 7900HT Fast RealTime PCR System was obtained from Applied Biosystems.

Western blot analysis. We prepared samples for western blotting as described in our previous study (43). Total protein extraction and BCA protein assay kits were both purchased from KeyGen (Nanjing, China). Polyvinylidene fluoride (PVDF) membranes were purchased from Millipore (Bedford, MA, USA). PRL-3 antibody [anti-PTP4A3 (ab50276)] was purchased from Abcam (Cambridge, UK) which recognizes human PRL-3 and does not cross-react with human PRL-1 and PRL-2. PRL-3 antibody (ab50276) was also used in previous studies (44-47). The following antibodies of PTEN, Akt, MMP-2, MMP-9, p-Akt and p-PTEN were also purchased from Abcam. The antibody for $\beta$-actin was purchased from Proteintech Group, Inc. (Wuhan, China). ECL western blotting analysis system was obtained from TransGen Biotech (Beijing, China).

Cell migration and invasion assays. Cell migration and invasion assays were performed as previously described (18).
Transwell cell culture chambers $(8.0-\mu \mathrm{m}$ pore polycarbonate membranes) were purchased from Becton-Dickinson (San Diego, CA, USA). The fluorescence microscope was purchased from Olympus (Center Valley, PA, USA) and was used to capture the images.

Statistical analysis. Each experiment was repeated at least three times. All of the data are presented as the mean \pm SD. Statistical analysis was performed using SPSS 19.0 computer software (SPSS, Inc., Chicago, IL, USA) and software program GraphPad Prism. Differences among variables were assessed by $\chi^{2}$ analysis, Student's t-test or ANOVA. In all cases, a value of $\mathrm{P}<0.05$ was accepted as significant.

\section{Results}

Overexpression of PRL-3 is inversely proportional to the low expression of PTEN in GC tissues. To investigate the relationship between PRL-3 and PTEN expression in GC tissues, we analyzed the expression of PRL-3 and PTEN in 21 specimens of normal gastric mucosa, 49 specimens of $\mathrm{GC}$ without peritoneal metastasis, and 23 specimens of GC with peritoneal metastasis by qRT-PCR and western blot analysis. PRL-3 was overexpressed in the GC samples with peritoneal metastasis when compared with the level in normal gastric mucosa both at the mRNA (data not shown) and the protein level (Fig. 1A and B). This indicates that high expression of PRL-3 is closely related to the tumor stage of GC. However, there was no significant statistical difference in the mRNA level of PTEN among the three types of gastric tissues, implying that the downregulation of PTEN expression was a post-transcriptional event. We also observed that weak to strong expression of PTEN protein was noted in GC with peritoneal metastasis, GC without peritoneal metastasis, and normal gastric mucosa by western blotting (Fig. 1C and D). The p-PTEN expression level was also lower in normal gastric mucosa than that noted in GC with peritoneal metastasis. Moreover, we conducted further analysis of the data by western blot analysis. It revealed that the p-PTEN and PTEN ratio was higher in GC cells when compared with the ratio in the normal gastric mucosal cells (Fig. 1E). Furthermore, we used an enzyme activity detection kit to detect the phosphatase activity of PRL-3. From these data, the enzyme activity of PRL-3 was more active in GC with peritoneal metastasis than that noted in the normal gastric mucosa (Fig. 1F). All of the data indicated that low expression of PTEN may be inversely correlated to both the high enzyme activity and the high expression of PRL-3. The high enzyme activity of PRL-3 may increase the phosphorylation level of PTEN.

PRL-3 is overexpressed is contrast to the low expression of $P T E N$ in GC cell lines. To explore the relationship between the expression of PTEN and PRL-3, we analyzed these levels in one normal human gastric mucosal cell line GES-1 and five GC cell lines of different metastatic potential (MGC803, MKN28, AGS, MKN45 and SGC7901) by qRT-PCR and western blotting. The expression level of PRL-3 in the five GC cell lines was much higher than that in the GES-1 cell line (Fig. 2A and B). The PTEN expression was lower in the five GC cell lines than that in normal gastric mucosal cell line at the mRNA and 
A

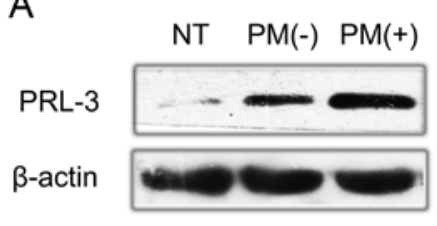

C
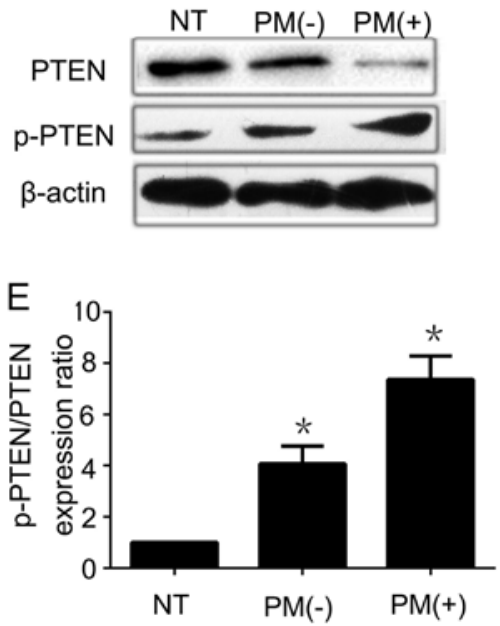
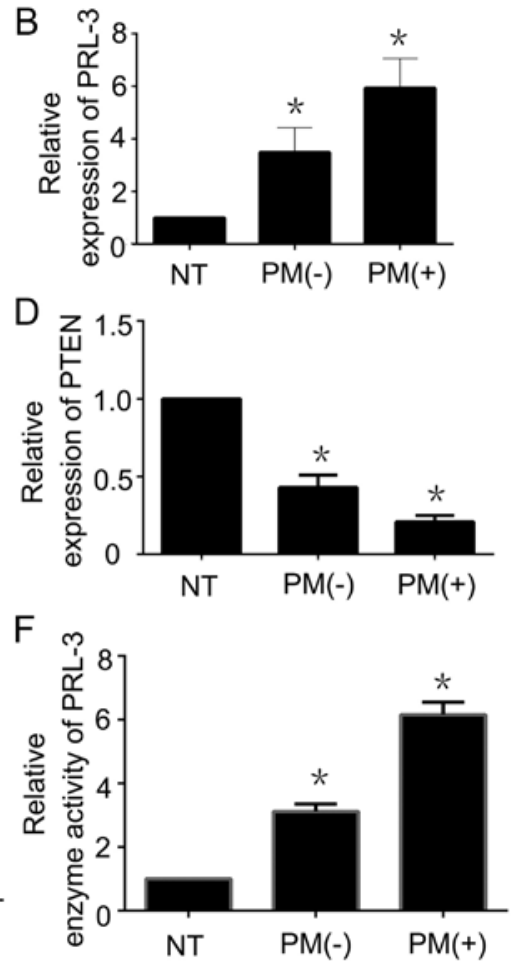

Figure 1. Overexpression of PRL-3 is inversely proportional to the low expression of PTEN in gastric cancer tissues. (A) PRL-3 was overexpressed in GC with peritoneal metastasis by western blotting. (B) Relative expression of PRL-3 protein in 21 specimens of normal gastric mucosa, 49 specimens of GC without peritoneal metastasis, and 23 specimens of GC with peritoneal metastasis was analyzed by western blot analysis. (C) PTEN was weakly expressed in GC with peritoneal metastasis when compared with that in the normal gastric mucosal tissues. p-PTEN expression was weakly expressed in the normal gastric mucosal tissues by western blotting. (D) Relative expression of PTEN protein in 21 specimens of normal gastric mucosa, 49 specimens of GC without peritoneal metastasis, and 23 specimens of GC with peritoneal metastasis was analyzed by western blot analysis. (E) Relative p-PTEN/PTEN protein expression ratio in 21 specimens of normal gastric mucosa, 49 specimens of GC without peritoneal metastasis, and 23 specimens of GC with peritoneal metastasis was analyzed by western blot analysis. (F) Relative enzyme activity of PRL-3 in 21 specimens of normal gastric mucosa, 49 specimens of GC without peritoneal metastasis, and 23 specimens of GC with peritoneal metastasis was analyzed by enzyme activity detection kit. Each bar shows the mean \pm standard deviation from three independent experiments; ${ }^{*} \mathrm{P}<0.05$ vs. the normal gastric mucosa tissues. NT, normal gastric mucosa tissues; PM(-), GC without peritoneal metastasis; PM(+), GC with peritoneal metastasis.

protein level (Fig. 2C and D). The p-PTEN/PTEN ratio was higher in GC cells than that in the normal gastric mucosal cell line (Fig. 2E). We also detected the enzyme activity level of PRL-3 by an enzyme activity detection kit. PRL-3 phosphatase activity was higher in the GC cell lines (Fig. 2F). The results indicated that PRL-3, through its phosphatase activity, led to the low expression of PTEN and phosphorylated it to facilitate the development of peritoneal metastasis by GC. As PRL-3 decreased the expression of PTEN and increased the ratio of p-PTEN/PTEN to a greater extent in the SGC7901 cells, we chose the SGC7901 GC cell line to perform the next experiment.

PRL-3 downregulates the expression of PTEN through its phosphatase activity. To further investigate whether or not PRL-3 through its enzyme activity downregulates PTEN, we constructed a wild-type plasmid EGFP-PRL-3 and a mutant-type plasmid EGFP-PRL-3 (D72A/C104S) without the enzyme activity of PRL-3. After transfecting the EGFP-PRL-3, EGFP-PRL-3 (D72A/C104S) and PLNCX vector (control empty plasmid) into SGC7901 cells and screening the stably expressed cell lines, we detected the phosphatase activity of PRL-3 using the enzyme activity detection kit. The wild-type with enzyme activity and the mutant-type without enzyme activity met our requirements. Next, we detected the expression of PRL-3 and PTEN by qRT-PCR and western blotting. There was no significant difference between the wild-type and the mutant-type in terms of PRL-3 expression, but both were higher than the control group (Fig. 3A). PTEN expression was lower in the wild-type than the level in the mutant-type, while the ratio of p-PTEN/PTEN was higher in the wildtype (Fig. 3A and B). This shows that high expression of PRL-3 downregulates the expression of PTEN and increases the ratio of p-PTEN/PTEN. Further analysis indicated that the higher enzyme activity of PRL-3 had an opposite relationship with the expression of PTEN. PRL-3 increased the ratio of $\mathrm{p}-\mathrm{PTEN} / \mathrm{PTEN}$ and promoted PTEN phosphorylation and inactivated it through its phosphatase activity. We also analyzed the migration and invasion abilities of the different groups by Transwell assay. The mutant-type had less ability to promote GC cell migration and invasion than the wildtype (Fig. 3C-E). All of the observations suggest that PRL-3 may downregulate PTEN and induce PTEN inactivity through its enzyme activity to promote GC cell metastasis.

Matrix metallopeptidases (MMP-2 and MMP-9) are involved in the invasion and migration of GC cells regulated by $P R L-3$. To dissect the mechanism of MMPs in PRL-3-promoted GC 

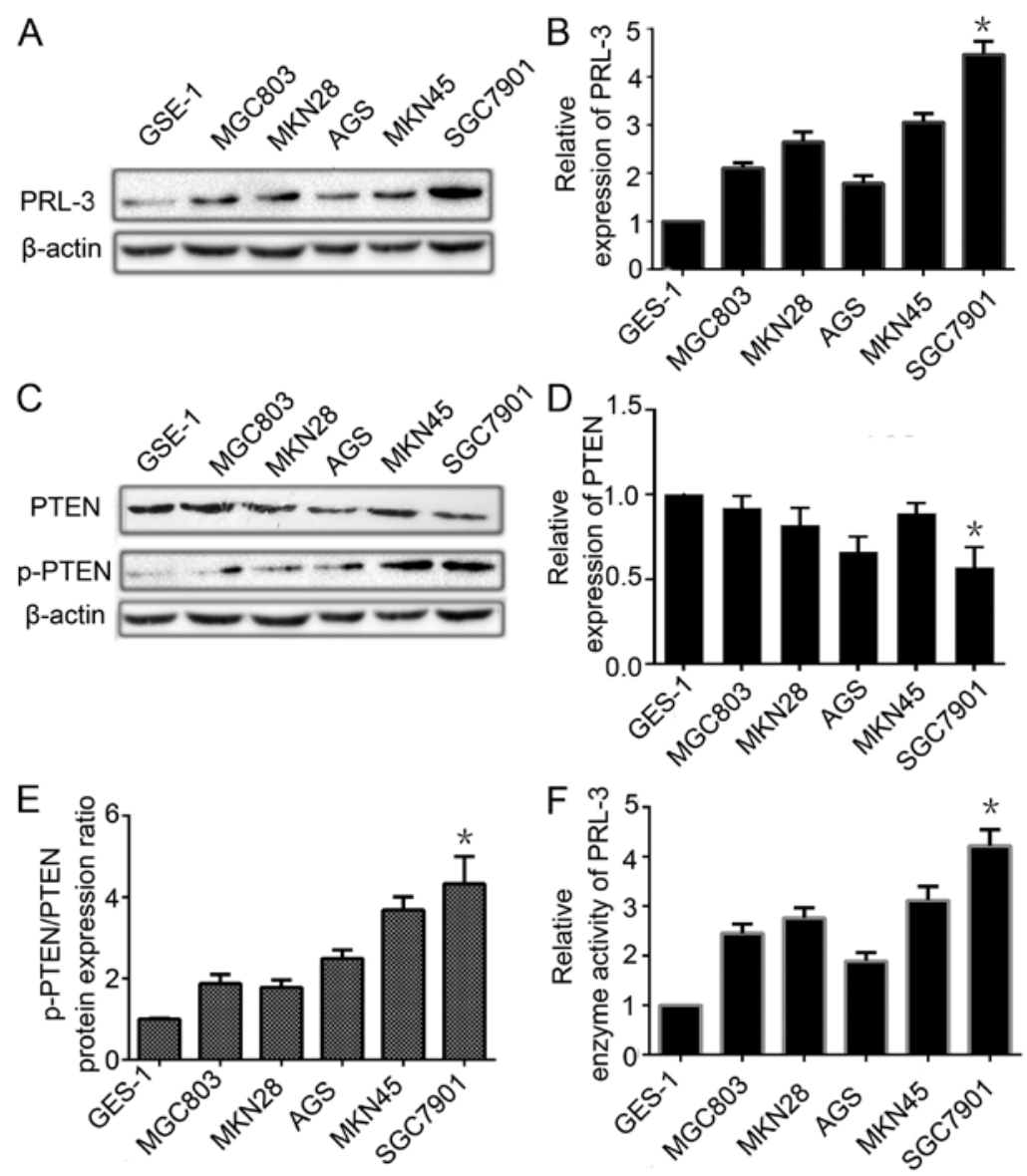

Figure 2. PRL-3 is overexpressed is contrast to the low expression of PTEN in gastric cancer cell lines. (A) The expression of PRL-3 in one normal human gastric mucosal cell line (GES-1) and five gastric cancer cell lines (MGC803, MKN28, AGS, MKN45 and SGC7901) was analyzed by western blotting. $\beta$-actin was used as an internal control. (B) Relative expression of PRL-3 in A was determined as a ratio to $\beta$-actin. (C) The expression of PTEN and p-PTEN in one normal human gastric mucosal cell line (GES-1) and five gastric cancer cell lines (MGC803, MKN28, AGS, MKN45 and SGC7901) was analyzed by western blotting. $\beta$-actin was used as an internal control. (D) Relative expression of PTEN in C was determined as a ratio to $\beta$-actin. (E) Relative p-PTEN/PTEN protein expression ratio in $\mathrm{C}$ was determined as a ratio to that in the GES-1 cells. (F) Relative enzyme activity of PRL-3 in different GC cell lines compared with the GES-1 cell line. Each bar shows the mean \pm standard deviation from three independent experiments; ${ }^{*} \mathrm{P}<0.05$ vs. the GES-1 cells.

cell migration and invasion, we further analyzed the expression of MMP-2 and MMP-9 in PRL-3 high expression and PRL-3 low expression groups by western blot analysis. The phosphatase activity of PRL-3 was lower in the PRL-3 low expression group than the SGC7901 cell group and PRL-3 high expression group as detected by the enzyme activity detection kit. The expression levels of MMP-2 and MMP-9 were consistently higher in the high group than that in the SGC7901 cells. When we silenced PRL-3 in the low expression group, the expression levels of MMP-2 and MMP-9 were reduced as expected (Fig. 4A-C). We also evaluated the invasion and metastasis in the different groups. Invasion and metastasis were significantly inhibited in the PRL-3 low expression group with low expression of MMP-2 and MMP-9 compared with the control group (Fig. 4D and E). To further confirm that PRL-3 promotes GC metastasis by upregulating the expression of MMP-2 and MMP-9, we added $20 \mu$ M MMP-2/MMP-9 inhibitor I into the different groups before performing the migration and invasion assays. The data showed that MMP-2/MMP-9 inhibitor I inhibited the PRL-3-promoted invasion and metastasis abilities of the SGC7901 cells (Fig. 4F and G). In conclusion, all the results indicated that PRL-3 promoted GC cell migration and invasion by upregulating the expression of MMP-2 and MMP-9.

The Akt pathway is involved in the upregulation of MMP-2 and MMP-9 and the invasion and migration of GC cells mediated by PRL-3. The PI3K/Akt pathway plays a critical role in the control of tumor cell growth, survival, progression, apoptosis, invasion and metastasis, and AKT is the central mediator of the PI3K/Akt pathway (48). Since researchers have reported that PRL-3 activates the PI3K/Akt pathway to promote cancer cell invasion and metastasis (30), we next detected the effect of PRL-3 expression on AKT activation in GC. PRL-3 increased the expression of Akt in the PRL-3 high expression group. When we knocked down PRL-3 by RNAi, the expression of Akt was also decreased (Fig. 5A and B). We also found that the p-Akt/Akt ratio was higher in the PRL-3 high expression group than that in the control group. Following knockdown of PRL-3 in the SGC7901 GC cell line, the ratio of p-Akt/Akt was also decreased (Fig. 5C). When we added the PI3K inhibitor LY294002 into the PRL-3 high expression group, the effects of PRL-3 on Akt, MMP-2, MMP-9 was blocked, with concomitant inhibition of invasion and metastasis (data not 


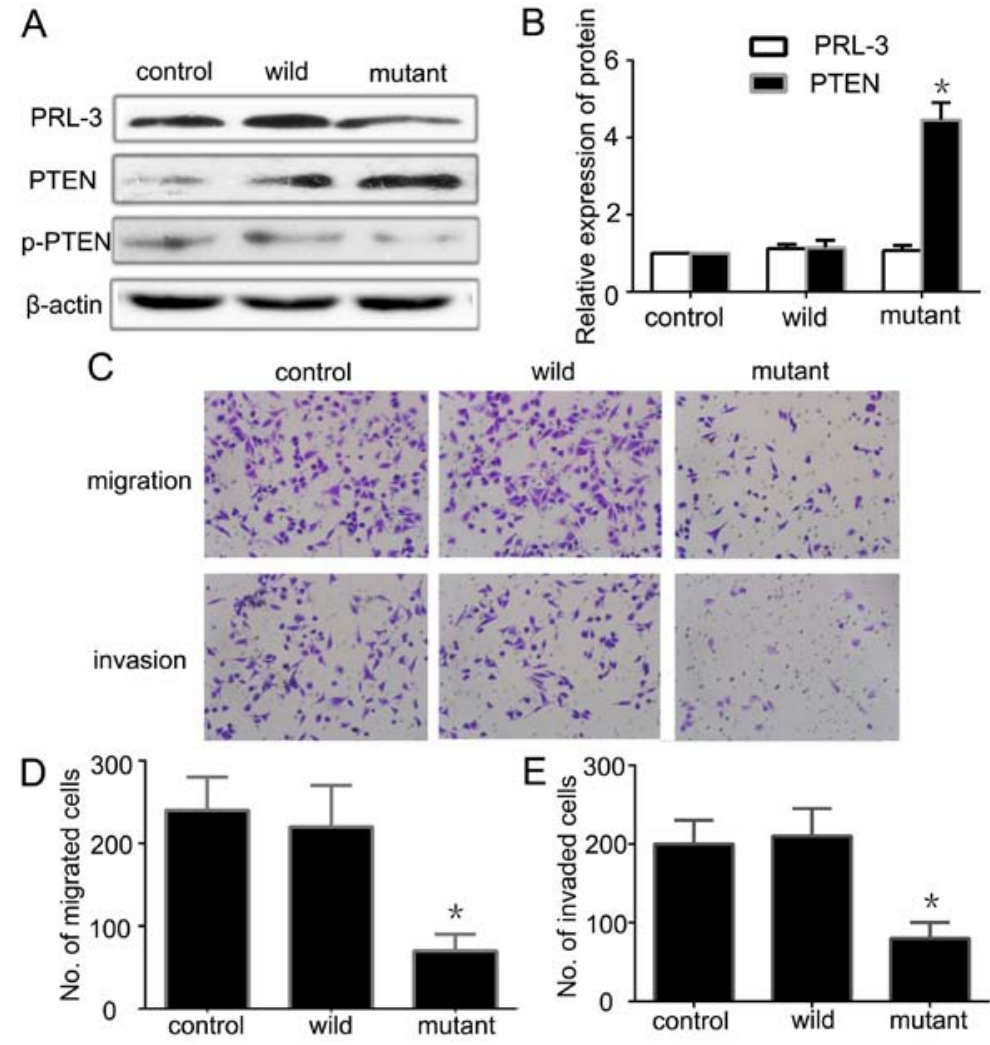

Figure 3. PRL-3 through its phosphatase activity downregulates the expression of PTEN. (A) The expression of PRL-3, PTEN and p-PTEN was analyzed in SGC7901 cancer cells (control), EGFP-PRL-3 cells (wild) and EGFP-PRL-3 (D72A/C104S) (mutant) cells by western blotting. (B) Relative expression of PRL-3 and PTEN in A was determined as a ratio to $\beta$-actin. (C) Cell migration and invasion were respectively analyzed. Original magnification, x200. (D and E) Each bar of the histogram indicates the number of stained cells. Vehicle group was used as a control. The experiments were repeated at least three times independently. Control, SGC7901 cancer cells; wild, EGFP-PRL-3 cells; mutant, EGFP-PRL-3 (D72A/C104S) cells. Each bar shows the mean \pm standard deviation from three independent experiments; ${ }^{\mathrm{P}}<0.05$ vs. the control group.

shown). All of the data show that PRL-3 upregulates the PI3K/ Akt signaling pathway to promote GC metastasis.

Ablation of PTEN expression restores the migration/invasion phenotype of PRL-3-silenced cancer cells. To further confirmed that the reduced migration and invasion were caused by the downregulation of PTEN by PRL-3, we next tested whether knockdown of PTEN in PRL-3-silenced cancer cells (the PRL-3 low expression group) reactivated the PI3K/Akt/MMP-2/MMP-9 signaling pathway and restored their migration/invasion phenotype. As expected, silencing of PTEN with RNAi in the PRL-3 low expression group restored the expression of PTEN and the Akt expression (Fig. 6A and B). Meanwhile, the ratio of $\mathrm{p}$-Akt/Akt and the expression of MMP-2 and MMP-9 were also restored (Fig. 6A and B). Furthermore, the cell migration and invasion ability was also partly restored in the PRL-3 low expression group when we transfected the PTEN-RNAi vector into the cells (Fig. 6C-F). These results suggested that PRL-3 decreases the expression of PTEN to promote GC migration and invasion.

\section{Discussion}

Phosphatase of regenerating liver-3 (PRL-3) is a metastasis-promoting phosphatase, which has been reported to promote the metastasis of a variety of cells, including gastric cancer (GC) SGC7901 $(14,49)$, colon cancer LoVo $(50)$, sali- vary adenoid cystic carcinoma SACC-83 (8) and lung cancer cells (42). In our previous study, we found that PRL-3 was highly expressed in GC (14), and miR-495 and miR-551a both act as tumor-suppressors by targeting the PRL-3 oncogene and inhibiting GC cell migration and invasion (18). In the present study, we also found that PRL-3 was overexpressed in GC tissues with peritoneal metastasis when compared with that in normal gastric tissues. High expression of PRL-3 was also found in GC cells that had a high degree of metastatic potential, as compared with corresponding normal cells. The results were in accordance with previous research studies $(16,51,52)$.

PTEN is a tumor-suppressor gene that occupies a key position in the regulation of cell growth, proliferation, apoptosis, mobility and signal transduction (53), while its expression and activity can be regulated at almost all levels: transcriptional, translational and post-translational (54). The function of PTEN is commonly lost in a large proportion of human cancers through somatic mutations, gene silencing or epigenetic mechanisms (55). In a previous study, researchers found that PRL-3 downregulated PTEN in the DLD-1 colorectal cell line (29). From our data, the PTEN mRNA expression was not statistically different between the GC tissues or the normal gastric mucosal tissues. However, the PTEN protein expression was lower in $\mathrm{GC}$ with peritoneal metastasis than that noted in normal gastric mucosal tissues. The present study also found that there was a higher level of PTEN phosphorylation in GC tissues than that noted in the normal gastric tissues. 

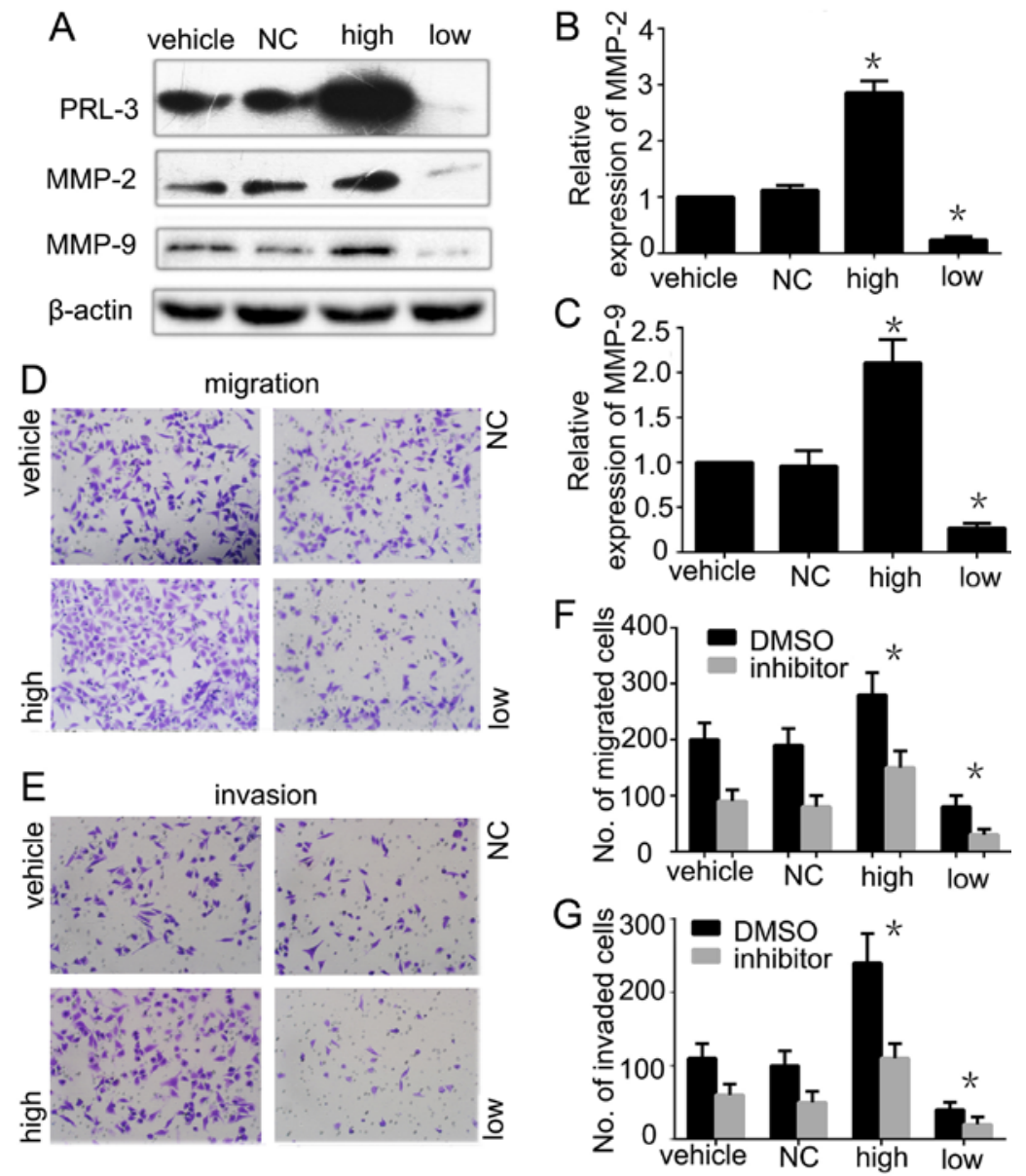

Figure 4. Matrix metallopeptidases (MMP-2 and MMP-9) are involved in the invasion and migration of gastric cancer cells regulated by PRL-3. (A) The expression levels of PRL-3, MMP-2 and MMP-9 were analyzed in SGC7901 cells (vehicle), SGC7901 cells transfected with control empty plasmid (NC), SGC7901/EGFP-PRL-3 cells (high) and SGC7901/PRL-3-RNAi cells (low) by western blotting. All groups were treated with Lipofectamine 2000 reagent. Relative expression of (B) MMP-2 and (C) MMP-9 in A, was determined as a ratio to $\beta$-actin. (D) Cell migration and (E) invasion were respectively analyzed by Transwell assays. Original magnification, x200. (F) Cell migration and (G) invasion were analyzed before or after inhibitor was added to each group. Each bar in the histogram indicates the number of stained cells. Vehicle group was used as a control. The experiments were repeated at least three times independently. Vehicle, SGC7901 cells; NC, SGC7901 transfected by control empty; high, plasmid SGC7901/EGFP-PRL-3 cells; low, SGC7901/PRL-3-RNAi cells; "P<0.05.
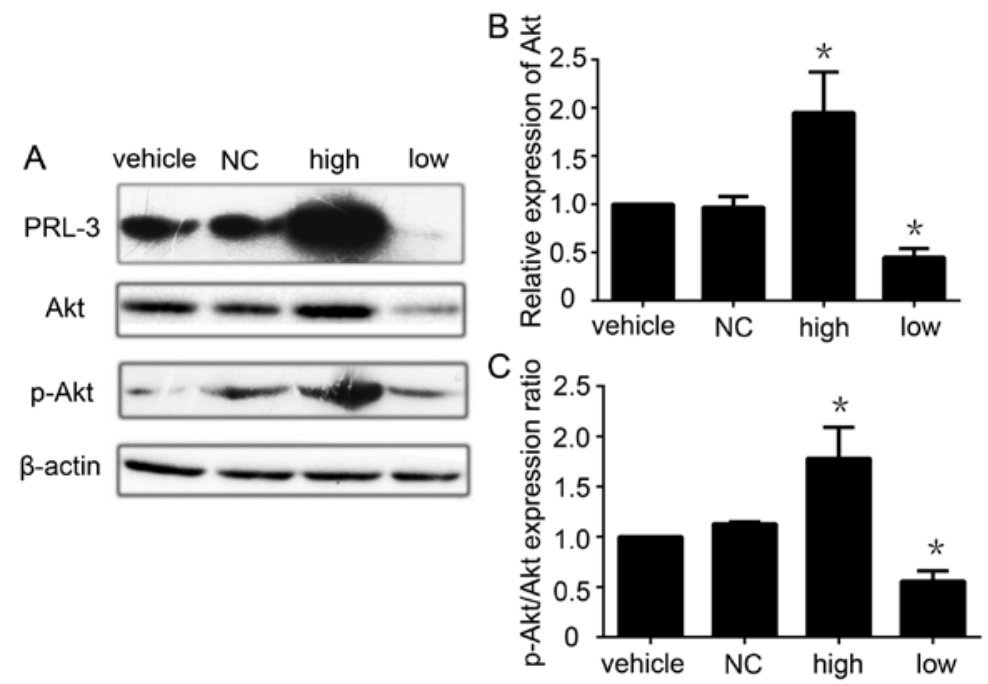

Figure 5. Akt pathway is involved in the upregulation of MMP-2 and MMP-9 and the invasion and migration of gastric cancer cells mediated by PRL-3. (A) The expression of PRL-3, Akt and p-Akt/Akt was analyzed in SGC7901 cells (vehicle), SGC7901 cells transfected with empty control (NC), plasmid SGC7901/EGFP-PRL-3 cells (high) and SGC7901PRL-3-RNAi cells (low) by western blotting. (B) Relative expression of Akt in A. (C) Relative p-Akt/Akt protein expression ratio in A was determined as a ratio to that in the vehicle group. Vehicle group was used as a control. The experiments were repeated at least three times independently. Vehicle, SGC7901 cells; NC, SGC7901 cells transfected by control empty; high, plasmid SGC7901/EGFP-PRL-3 cells; low, SGC7901/PRL-3-RNAi cells. Each bars shows the mean \pm standard deviation from three independent experiments; "P<0.05. 

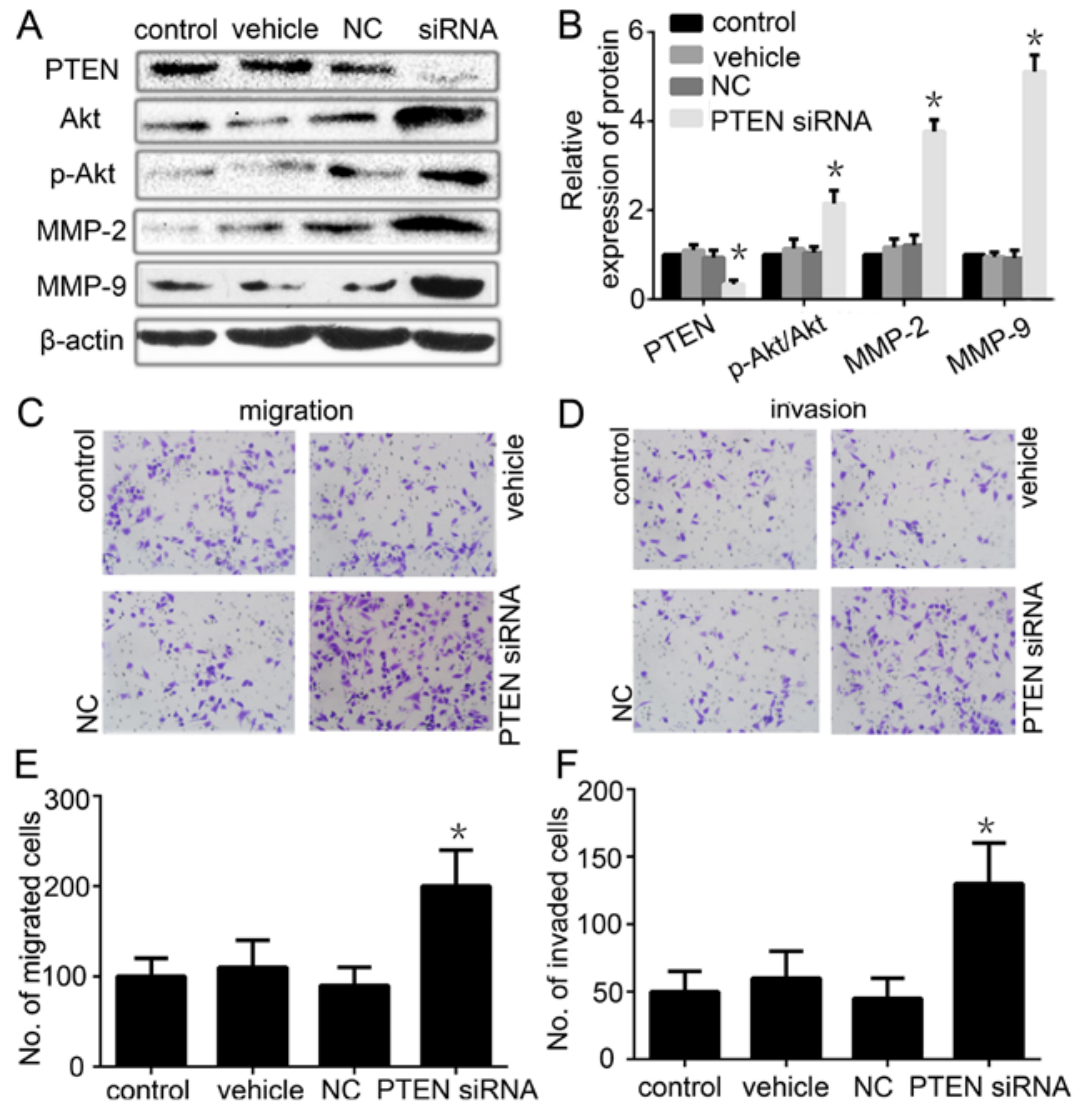

Figure 6. Ablation of PTEN expression restores the migration/invasion phenotype in PRL-3-silenced cancer cells. (A) The expression of PTEN, Akt, p-Akt, MMP-2 and MMP-9 was analyzed by western blotting after PTEN RNAi was transfected into the PRL-3 stable low expression cells. (B) Relative expression of PTEN, Akt, p-Akt, MMP-2 and MMP-9 in A was determined as a ratio to $\beta$-actin. (C) Cell migration and (D) invasion were respectively analyzed. Original magnification, x200. (E) Migration and (F) invasion cell numbers are indicated in the bar graph. Each value is the number of stained cells. Control, PRL-3 low expression SGC7901 cells; vehicle, PRL-3 low expression SGC7901 cells (only used Lipofectamine 2000 reagent); NC, PRL-3 low expression SGC7901 cells transfected with empty control plasmid; siRNA, PRL-3 low expression SGC7901 cells transfected with PTEN siRNA. Control and vehicle groups were used as a control. The experiments were repeated at least three times independently. Each bar shows the mean \pm standard deviation from three independent experiments; ${ }^{*} \mathrm{P}<0.05$.

It showed that the downregulation of PTEN expression was a post-transcriptional regulation event. We also found the same phenomenon in the GC cell lines and the gastric mucosa cell line GES-1. The enzyme activity of PRL-3 was higher in GC with peritoneal metastasis, as well as in the GC cell lines, than that in the normal gastric mucosa and the gastric mucosal cell line GES-1. All of the data indicate that PRL-3 may downregulate the expression of PTEN and induce PTEN phosphorylation through its enzyme activity.

To further explore the relationship between the enzyme activity of PRL-3 and downregulation of PTEN expression and high level phosphorylation of PTEN, we constructed a wild-type plasmid EGFP-PRL-3 and a mutant-type plasmid EGFP-PRL-3 (D72A/C104S) without enzyme activity and transfected the plasmids into the SGC7901 cells. PRL-3 with high enzyme activity significantly downregulated PTEN expression and induced PTEN phosphorylation compared to the no enzyme activity cells. All of the data indicated that PRL-3 downregulated the expression of PTEN and induced PTEN phosphorylation to inactivite it through its phosphatase activities. Meanwhile, silencing of PTEN with RNAi in PRL-3-silenced cells restored the cell migration and invasion ability, which further demonstrated that PRL-3 through its phosphatase activity downregulated PTEN, which led to GC cell migration and invasion. However, the mechanism behind the PRL-3 downregulation of PTEN remains unknown. As a phosphatase of PRL-3, it is almost not possible to induce PTEN phosphorylation. We speculate that, on one hand, PRL-3 may decrease the expression of PTEN. On the other hand, PRL-3 may through its enzyme activity dephosphorylate a certain kinase and improve its enzyme activity, and then the kinase phosphorylates PTEN and makes it inactive. All of the speculations need further validation.

Metastasis is a key step in cancer progression that indicates a more advanced stage and a poorer prognosis. Tumor cells escape from the primary site and migrate into the circulatory system which are important steps in cancer metastasis (56). Extracellular matrix degradation plays an important role in tumor invasion and metastasis, which is mainly mediated by MMP-2 and MMP-9 (57). In our previous study, we found that overexpression of PRL-3 promoted GC invasion and metastasis (14). Thus, it was necessary to investigate whether the involvement of PRL-3 in invasion and migration is also correlated with MMP-2 and MMP-9 in GC. The data indicated that PRL-3 increased the expression of MMP-2/MMP-9 in the PRL-3 high expression group, while silencing of PRL-3 with RNAi resulted in reduction of MMP-2/MMP-9 expression. Moreover, MMP-2/MMP-9 inhibitor I obstructed the 
migration and invasion abilities in the PRL-3 high expression group. These data suggest that PRL-3 may upregulate MMP-2 and MMP-9 expression leading to GC metastasis. Of course, it may be more appropriate to detect the expression of MMP-2/MMP-9 in secreted media (35), and not only in cellular lysates. This is a limitation of the present study.

$\mathrm{PI} 3 \mathrm{~K}$ signaling is a crucial regulator of many essential cellular processes, including cell growth, metabolism, survival, metastasis and resistance to chemotherapy (58). PI3K activity is regulated by the lipid phosphatase and tensin homolog (PTEN), a tumor-suppressor gene that encodes a lipid phosphatase that downregulates the PI3K signal by converting PIP3 back to PIP2 (59). Loss of PTEN function, as well as PI3K activation, results in accumulation of $\mathrm{PI} 3 \mathrm{P}$ triggering the activation of its downstream effection (60). In the present study, we found that PRL-3 downregulated PTEN, phosphorylated PTEN and inactivated it. However, whether the PI3K/Akt signaling pathway is also involved in the promotion of GC metastasis by PRL-3, also needs to be investigated. In our research, PRL-3 overexpression in the PRL-3 high expression group increased the expression of Akt and increased the ratio of p-Akt/Akt, accompanied by upregulation of MMP-2/MMP-9 expression and promoted the GC cell migration and invasion. The expression levels of Akt, MMP-2 and MMP-9 were blocked by the PI3K inhibitor LY294002. These data suggest that PRL-3 may signal through the PI3K/Akt pathway to upregulate MMP-2/ MMP-9 leading to migration and invasion of GC.

In summary, we identified that PRL-3 downregulates PTEN and induces PTEN phosphorylation then activates, at least partly, the PI3K/Akt signaling pathway, as well as upregulation of MMP-2 and MMP-9, to promote the peritoneal metastasis of GC. However, whether or not PRL-3 directly downregulates PTEN protein remains unknown, and warrants further investigation. In conclusion, the present study revealed the potential mechanism of PRL-3-mediate GC invasion and metastasis, which will provide new strategies for the prevention and treatment of GC peritoneal metastasis.

\section{Acknowledgements}

The present study was supported by the National Science Foundation of China (no. 81160304/81360362), the Education Department of Jiangxi Province Science and Technology Research Projects (no. GJJ13126), the Training Program for Young Scientists of Jiangxi Province (no. 20133BCB23028), and the Graduate Student Innovation Special Fund Project of Nanchang University (no. cx2015189). We thank the Department of Gastrointestinal Surgery of The First Affiliated Hospital of Nanchang University for providing human gastric tissue samples. We also thank The Key Laboratory of Basic Pharmacology of Nanchang University and the Department of Histology and Embryology of Medical College of Nanchang University for technical assistance in the experiments.

\section{References}

1. Globocan 2012: Estimated Cancer Incidence, Mortality and Prevalence Worldwide in 2012. http://globocan.iarc.fr/ Pages/fact_sheets_cancer.aspx. Accessed March 31, 2014.

2. Siegel RL, Miller KD and Jemal A: Cancer statistics, 2015. CA Cancer J Clin 65: 5-29, 2015.
3. De Vita F, Vecchione L, Galizia G, Di Martino N, Fabozzi T, Catalano G, Ciardiello F and Orditura M: Perspectives in adjuvant therapy of gastric cancer. Oncology 77 (Suppl 1): S38-S42, 2009.

4. Kagawa S, Shigeyasu K, Ishida M, Watanabe M, Tazawa H, Nagasaka T, Shirakawa Y and Fujiwara T: Molecular diagnosis and therapy for occult peritoneal metastasis in gastric cancer patients. World J Gastroenterol 20: 17796-17803, 2014.

5. Bando E, Yonemura Y, Takeshita Y, Taniguchi K, Yasui T, Yoshimitsu Y, Fushida S, Fujimura T, Nishimura G and Miwa K: Intraoperative lavage for cytological examination in 1,297 patients with GC. Am J Surg 178: 256-262, 1999.

6. Turaga KK, Gamblin TC and Pappas S: Surgical treatment of peritoneal carcinomatosis from gastric cancer. Int J Surg Oncol 2012: 405652, 2012.

7. Bessette DC, Qiu D and Pallen CJ: PRL PTPs: Mediators and markers of cancer progression. Cancer Metastasis Rev 27: 231-252, 2008.

8. Dong Q, Ding X, Chang B, Wang $\mathrm{H}$ and Wang A: PRL-3 promotes migration and invasion and is associated with poor prognosis in salivary adenoid cystic carcinoma. J Oral Pathol Med 45: 111-118, 2016.

9. Kato H, Semba S, Miskad UA, Seo Y, Kasuga M and Yokozaki H: High expression of $P R L-3$ promotes cancer cell motility and liver metastasis in human colorectal cancer: A predictive molecular marker of metachronous liver and lung metastases. Clin Cancer Res 10: 7318-7328, 2004.

10. Guo K, Li J, Tang JP, Koh V, Gan BQ and Zeng Q: Catalytic domain of PRL-3 plays an essential role in tumor metastasis: Formation of PRL-3 tumors inside the blood vessels. Cancer Biol Ther 3: 945-951, 2004

11. Wu X, Zeng H, Zhang X, Zhao Y, Sha H, Ge X, Zhang M, Gao X and Xu Q: Phosphatase of regenerating liver-3 promotes motility and metastasis of mouse melanoma cells. Am J Pathol 164: 2039-2054, 2004.

12. Dai N, Lu AP, Shou CC and Li JY: Expression of phosphatase regenerating liver 3 is an independent prognostic indicator for gastric cancer. World J Gastroenterol 15: 1499-1505, 2009.

13. Zeng Q, Dong JM, Guo K, Li J, Tan HX, Koh V, Pallen CJ, Manser E and Hong W: PRL-3 and PRL-1 promote cell migration, invasion, and metastasis. Cancer Res 63: 2716-2722, 2003.

14. Li ZR, Wang Z, Zhu BH, He YL, Peng JS, Cai SR, Ma JP and Zhan WH: Association of tyrosine PRL-3 phosphatase protein expression with peritoneal metastasis of GC and prognosis. Surg Today 37: 646-651, 2007.

15. Xing X, Lian S, Hu Y, Li Z, Zhang L, Wen X, Du H, Jia Y, Zheng Z, Meng L, et al: Phosphatase of regenerating liver-3 (PRL-3) is associated with metastasis and poor prognosis in GC. J Transl Med 11: 309-309, 2013.

16. Miskad UA, Semba S, Kato H and Yokozaki H: Expression of PRL-3 phosphatase in human gastric carcinomas: Close correlation with invasion and metastasis. Pathobiology 71: 176-184, 2004.

17. Wang Z, Cai SR, He YL, Zhan WH, Chen CQ, Cui J, Wu WH, $\mathrm{Wu} \mathrm{H}$, Song W, Zhang $\mathrm{CH}$, et al: High expression of PRL-3 can promote growth of gastric cancer and exhibits a poor prognostic impact on patients. Ann Surg Oncol 16: 208-219, 2009.

18. Li Z, Cao Y, Jie Z, Liu Y, Li Y, Li J, Zhu G, Liu Z, Tu Y, Peng G, et al: miR-495 and miR-551a inhibit the migration and invasion of human gastric cancer cells by directly interacting with $P R L-3$. Cancer Lett 323: 41-47, 2012.

19. Waite KA and Eng C: Protean PTEN: Form and function. Am J Hum Genet 70: 829-844, 2002.

20. Bermúdez Brito M, Goulielmaki E and Papakonstanti EA: Focus on PTEN regulation. Front Oncol 5: 166, 2015.

21. Ye B, Jiang LL, Xu HT, Zhou DW and Li ZS: Expression of $\mathrm{PI} 3 \mathrm{~K} / \mathrm{AKT}$ pathway in gastric cancer and its blockade suppresses tumor growth and metastasis. Int J Immunopathol Pharmacol 25: 627-636, 2012.

22. Myers MP, Pass I, Batty IH, Van der Kaay J, Stolarov JP, Hemmings BA, Wigler MH, Downes CP and Tonks NK: The lipid phosphatase activity of PTEN is critical for its tumor supressor function. Proc Natl Acad Sci USA 95: 13513-13518, 1998.

23. Stambolic V, Suzuki A, de la Pompa JL, Brothers GM, Mirtsos C, Sasaki T, Ruland J, Penninger JM, Siderovski DP and Mak TW: Negative regulation of $\mathrm{PKB} / \mathrm{Akt}$-dependent cell survival by the tumor suppressor PTEN. Cell 95: 29-39, 1998.

24. Cully M, You H, Levine AJ and Mak TW: Beyond PTEN mutations: The PI3K pathway as an integrator of multiple inputs during tumorigenesis. Nat Rev Cancer 6: 184-192, 2006. 
25. Wadhwa R, Song S, Lee JS, Yao Y, Wei Q and Ajani JA: Gastric cancer-molecular and clinical dimensions. Nat Rev Clin Oncol 10: 643-655, 2013.

26. Li NA, Wang W, Xu B and Gong H: miR-196b regulates gastric cancer cell proliferation and invasion via $\mathrm{PI} 3 \mathrm{~K} / \mathrm{AKT} / \mathrm{mTOR}$ signaling pathway. Oncol Lett 11: 1745-1749, 2016.

27. Akrami H, Mahmoodi F, Havasi S and Sharifi A: PlGF knockdown inhibited tumor survival and migration in gastric cancer cell via PI3K/Akt and p38MAPK pathways. Cell Biochem Funct 34: 173-180, 2016.

28. Sun L, Liu L, Liu X, Wang Y, Li M, Yao L, Yang J, Ji G, Guo C, Pan Y, et al: MGr1-Ag/37LRP induces cell adhesion-mediated drug resistance through FAK/PI3K and MAPK pathway in gastric cancer. Cancer Sci 105: 651-659, 2014.

29. Wang H, Quah SY, Dong JM, Manser E, Tang JP and Zeng Q: PRL-3 down-regulates PTEN expression and signals through PI3K to promote epithelial-mesenchymal transition. Cancer Res 67: 2922-2926, 2007.

30. Wang H, Vardy LA, Tan CP, Loo JM, Guo K, Li J, Lim SG, Zhou J, Chng WJ, Ng SB, et al: PCBP1 suppresses the translation of metastasis-associated PRL-3 phosphatase. Cancer Cell 18: 52-62, 2010.

31. Fang XY, Song R, Chen W, Yang YY, Gu YH, Shu YQ, Wu XD, Wu XF, Sun Y, Shen Y, et al: PRL-3 promotes the malignant progression of melanoma via triggering dephosphorylation and cytoplasmic localization of NHERF1. J Invest Dermatol 135 2273-2282, 2015.

32. Chaffer CL and Weinberg RA: A perspective on cancer cell metastasis. Science 331: 1559-1564, 2011.

33. Brinckerhoff CE and Matrisian LM: Matrix metalloproteinases: A tail of a frog that became a prince. Nat Rev Mol Cell Biol 3: 207-214, 2002.

34. Peng L, Xing X, Li W, Qu L, Meng L, Lian S, Jiang B, Wu J and Shou C: PRL-3 promotes the motility, invasion, and metastasis of LoVo colon cancer cells through PRL-3-integrin beta1-ERK1/2 and-MMP2 signaling. Mol Cancer 8: 110, 2009.

35. Ye Z, Al-Aidaroos AQ, Park JE, Yuen HF, Zhang SD, Gupta A, Lin Y, Shen HM and Zeng Q: PRL-3 activates mTORC1 in cancer progression. Sci Rep 5: 17046, 2015.

36. Li WJ, Xing XF, Qu LK, Meng L and Shou CC: Construction and expression of PRL-3 plasmid with C104S point mutation and CAAX deletion. Beijing Da Xue Xue Bao 41: 516-520, 2009 (In Chinese)

37. Pascaru M, Tanase C, Vacaru AM, Boeti P, Neagu E, Popescu I and Szedlacsek SE: Analysis of molecular determinants of PRL-3. J Cell Mol Med 13: 3141-3150, 2009.

38. Kozlov G, Cheng J, Ziomek E, Banville D, Gehring K and Ekiel I: Structural insights into molecular function of the metastasis-associated phosphatase PRL-3. J Biol Chem 279: 11882-11889, 2004.

39. McParland V, Varsano G, Li X, Thornton J, Baby J, Aravind A, Meyer C, Pavic K, Rios P and Köhn M: The metastasis-promoting phosphatase PRL-3 shows activity toward phosphoinositides. Biochemistry 50: 7579-7590, 2011

40. Matter WF, Estridge T, Zhang C, Belagaje R, Stancato L, Dixon J, Johnson B, Bloem L, Pickard T, Donaghue M, et al: Role of PRL-3, a human muscle-specific tyrosine phosphatase, in angiotensin-II signaling. Biochem Biophys Res Commun 283 1061-1068, 2001.

41. Fiordalisi JJ, Keller PJ and Cox AD: PRL tyrosine phosphatases regulate rho family GTPases to promote invasion and motility. Cancer Res 66: 3153-3161, 2006.

42. Jian M, Nan L, Guocheng J, Qingfu Z, Xueshan Q and Enhua W: Downregulating PRL-3 inhibit migration and invasion of lung cancer cell via RhoA and mDial. Tumori 98: 370-376, 2012.

43. Li Z, Zhan W, Wang Z, Zhu B, He Y, Peng J, Cai S and Ma J: Inhibition of PRL-3 gene expression in gastric cancer cell line SGC7901 via microRNA suppressed reduces peritoneal metastasis. Biochem Biophys Res Commun 348: 229-237, 2006.
44. Liu Y, Zheng P, Liu Y, Ji T, Liu X, Yao S, Cheng X, Li Y, Chen L, Xiao Z, et al: An epigenetic role for PRL-3 as a regulator of H3K9 methylation in colorectal cancer. Gut 62: 571-581, 2013.

45. Molleví DG, Aytes A, Padullés L, Martínez-Iniesta M, Baixeras N, Salazar R, Ramos E, Figueras J, Capella G and Villanueva A: PRL-3 is essentially overexpressed in primary colorectal tumours and associates with tumour aggressiveness. Br J Cancer 99: 1718-1725, 2008.

46. Mayinuer A, Yasen M, Mogushi K, Obulhasim G, Xieraili M, Aihara A, Tanaka S, Mizushima H, Tanaka H and Arii S: Upregulation of protein tyrosine phosphatase type IVA member 3 (PTP4A3/PRL-3) is associated with tumor differentiation and a poor prognosis in human hepatocellular carcinoma. Ann Surg Oncol 20: 305-317, 2013.

47. Khapare N, Kundu ST, Sehgal L, Sawant M, Priya R, Gosavi P, Gupta N, Alam H, Karkhanis M, Naik N, et al: Plakophilin3 loss leads to an increase in PRL3 levels promoting K8 dephosphorylation, which is required for transformation and metastasis. PLoS One 7: e38561, 2012

48. Zhang S, Zhang Y, Zhuang Y, Wang J, Ye J, Zhang S, Wu J, Yu K and Han Y: Matrine induces apoptosis in human acute myeloid leukemia cells via the mitochondrial pathway and Akt inactivation. PLoS One 7: e46853, 2012.

49. Bessette DC, Wong PC and Pallen CJ: PRL-3: A metastasis-associated phosphatase in search of a function. Cells Tissues Organs 185: 232-236, 2007.

50. Xu H, Lai W, Zhang Y, Liu L, Luo X, Zeng Y, Wu H, Lan Q and Chu Z: Tumor-associated macrophage-derived IL-6 and IL-8 enhance invasive activity of LoVo cells induced by PRL-3 in a KCNN4 channel-dependent manner. BMC Cancer 14: 330, 2014

51. Miskad UA, Semba S, Kato H, Matsukawa Y, Kodama Y, Mizuuchi E, Maeda N, Yanagihara K and Yokozaki H: High $P R L-3$ expression in human gastric cancer is a marker of metastasis and grades of malignancies: An in situ hybridization study. Virchows Archiv 450: 303-310, 2007.

52. Wang Z, He YL, Cai SR, Zhan WH, Li ZR, Zhu BH, Chen CQ, Ma JP, Chen ZX, Li W, et al: Expression and prognostic impact of PRL-3 in lymph node metastasis of gastric cancer: Its molecular mechanism was investigated using artificial microRNA interference. Int J Cancer 123: 1439-1447, 2008.

53. Xu W, Yang Z, Zhou SF and Lu N: Posttranslational regulation of phosphatase and tensin homolog (PTEN) and its functional impact on cancer behaviors. Drug Des Devel Ther 8: 1745-1751, 2014.

54. Bononi A and Pinton P: Study of PTEN subcellular localization. Methods 77-78: 92-103, 2015.

55. Milella M,Falcone I, Conciatori F, Cesta Incani U,Del Curatolo A, Inzerilli N, Nuzzo CM, Vaccaro V, Vari S, Cognetti F, et al: PTEN: Multiple functions in human malignant tumors. Front Oncol 5: 24, 2015.

56. Su Z, Yang Z, Xu Y, Chen Y and Yu Q: Apoptosis, autophagy, necroptosis, and cancer metastasis. Mol Cancer 14: 48, 2015.

57. Khasigov PZ, Podobed OV, Gracheva TS, Salbiev KD, Grachev SV and Berezov TT: Role of matrix metalloproteinases and their inhibitors in tumor invasion and metastasis. Biochemistry 68: 711-717, 2003.

58. Willems L, Tamburini J, Chapuis N, Lacombe C, Mayeux P and Bouscary D: PI3K and mTOR signaling pathways in cancer: New data on targeted therapies. Curr Oncol Rep 14: 129-138, 2012.

59. Ortega-Molina A and Serrano M: PTEN in cancer, metabolism, and aging. Trends Endocrinol Metab 24: 184-189, 2013.

60. Carnero A, Blanco-Aparicio C, Renner O, Link W and Leal JF: The PTEN/PI3K/AKT signalling pathway in cancer, therapeutic implications. Curr Cancer Drug Targets 8: 187-198, 2008. 